\title{
Miniaturized Multi-Band Antenna via Element Collocation
}

Ryan P. Martin

\section{What is the challenge?}

Although much research has been performed on a driven element parasitically loaded by another element shorted to ground for dual frequency operation, the novel concept of two or more coplanar-driven antennas in close proximity designed for multiple frequency operation has not been represented in the literature. Since each higher frequency antenna is built into the lower frequency elements, the largest element controls the structure's total size. Furthermore, by using the self-resonant frequency inherent in reactive elements due to device packaging, the aperture of each antenna, due to a low insertion loss path at the frequency of the larger element, will include that of all smaller radiators. This configuration provides a large standing wave ratio at the shorter wavelengths via several series capacitiveinductive connections. Therefore, each antenna element provides the required surface area for the frequency of operation while being isolated from the larger radiators. For this study, a dual $2.45 / 5.8 \mathrm{GHz}$ microstrip patch encompasses a small surface area of 9 square inches and provides circularly polarized electromagnetic radiation in excess of $6 \mathrm{dBi}$. This concept can be extrapolated to include additional radiators or may be scaled to other frequencies of interest. If a separate antenna were to be used for each frequency range, the overall device size would increase due to the presence of multiple apertures corresponding to the tunice size would increas each antenna element to circumvent parasitic loading of an antenna on its neighbor.

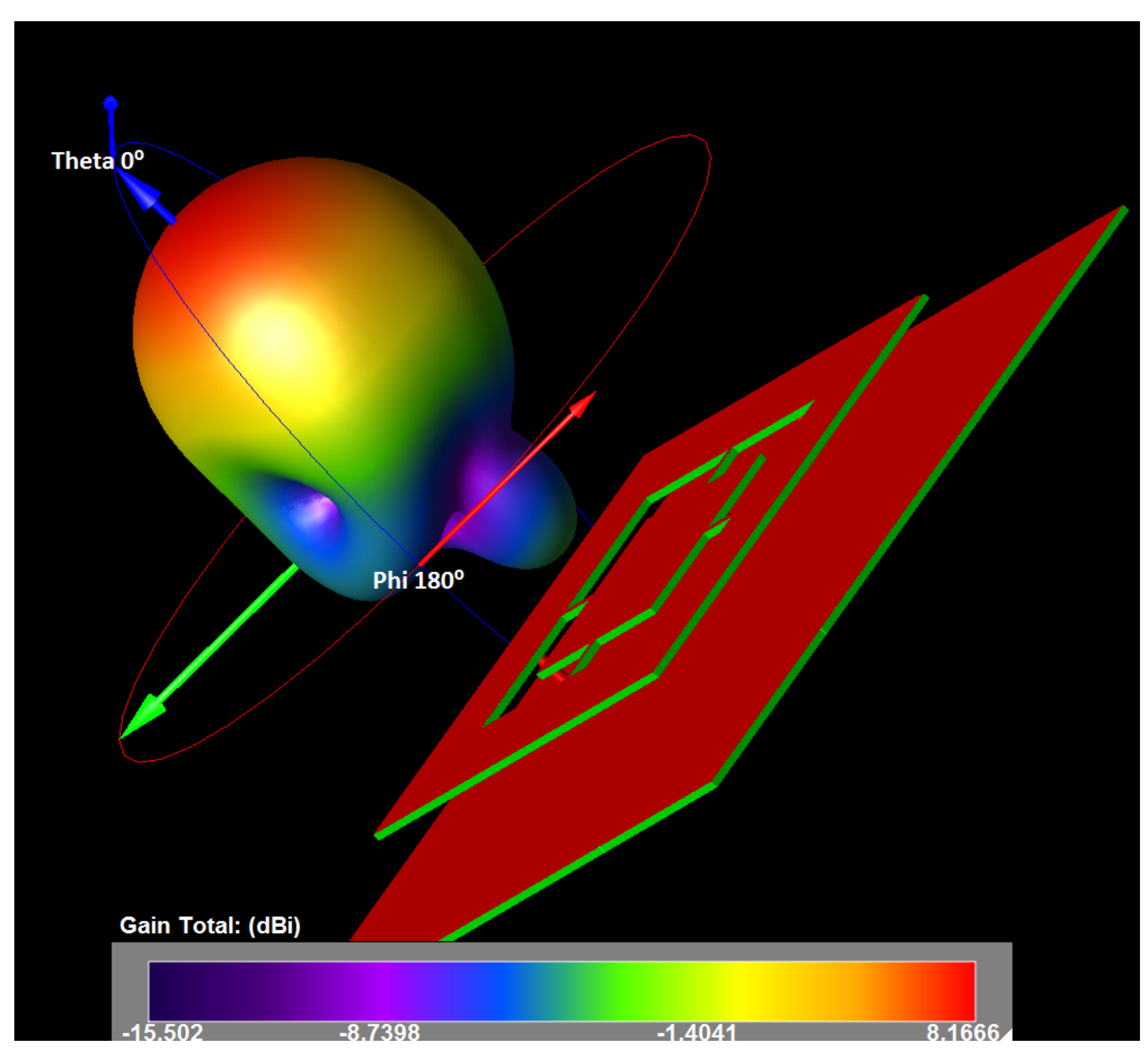

Three-dimensional,
$2.45 \mathrm{GHz}$, right-hand

circularly polarized

radiation pattern

superimposed
adjacent to the

coplanar $\mathrm{S}(2.45 \mathrm{GHz})$

coplanar $5(2.45 \mathrm{GHz})$
and $\mathrm{C}(5.8 \mathrm{GHz})$ band

radiators illustrates
excellent antenna

directivity with $60^{\circ}$

half-power beam
width. The antenn

will receive maximum
signal in the direction

normal to the antenna

region).

\section{What is our innovation?}

The resonant frequency of a microstrip patch antenna may be reduced through the addition of slots in the radiating element. Expanding upon this concept in favor of a significant reduction in the tuned width of the radiator, nearly $60 \%$ of the antenna metallization is removed, as seen in the top view of the antenna's radiating element (shown in red, below, left). To facilitate an increase in the gain of the antenna, the radiator is suspended over the ground plane (green) by an air substrate at a height of 0.250 " while being mechanically supported by 0.030 " thick Rogers R04003 laminate in the same profile as the element.

Although the entire surface of the antenna (red) provides $2.45 \mathrm{GHz}$ operation with insignificant negative effects on performance after material removal, the smaller square microstrip in the middle must be isolated from the additional aperture in order to afford higher frequency operation. A low insertion loss path centered at $2.45 \mathrm{GHz}$ may simultaneously provide considerable attenuation at additional frequencies through the implementation of a series-parallel, resonant reactive path. However, an inductive reactance alone will not permit lower frequency energy to propagate across the intended discontinuity. alone will not permit lower frequency energy to propagate across the intended disconting
To mitigate this, a capacitance is introduced in series with the inductor, generating a resonance at $2.45 \mathrm{GHz}$ with minimum forward transmission loss. Four of these reactive pairs are placed between the coplanar elements as shown. Therefore, the aperture of the lower-frequency outer segment includes the smaller radiator while the higher frequency section is isolated from the additional material.

In order to avoid cross-polarization losses due to the orientation of a transmitter or receiver in reference to the antenna, circular polarization is realized by a quadrature coupler for each collocated antenna as seen in the bottom view of the antenna (right). To generate electromagnetic radiation concentrically rotating about the direction of propagation, ideally one-half of the power must be delivered to the output of each branch with a phase shift of 90 degrees and identical amplitude. Due to this, each arm of the coupler is spaced $\lambda / 4$ wavelength apart.
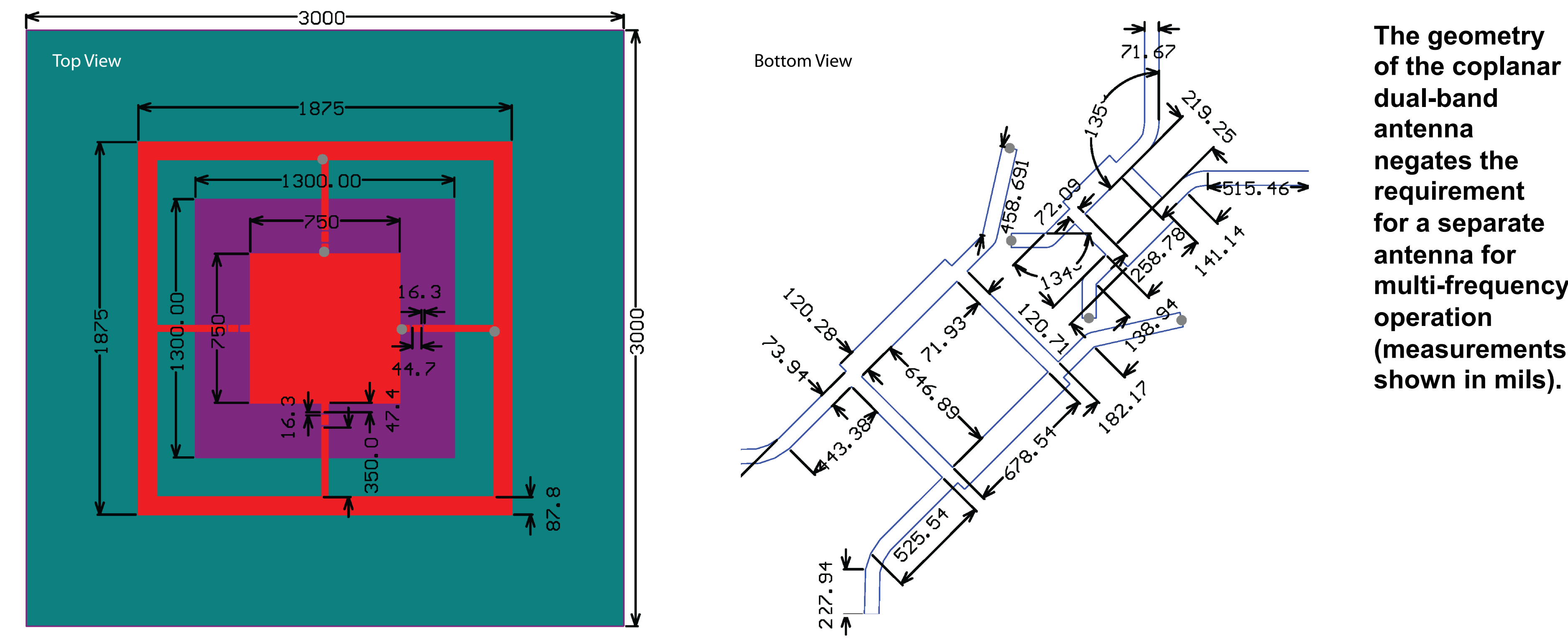

\section{What have we learned so far?}

As seen in the image below, the resonance at $5.8 \mathrm{GHz}$ of a $12 \mathrm{nH}$ inductor with its parasitic self-capacitance presents isolation in excess of $40 \mathrm{~dB}$.
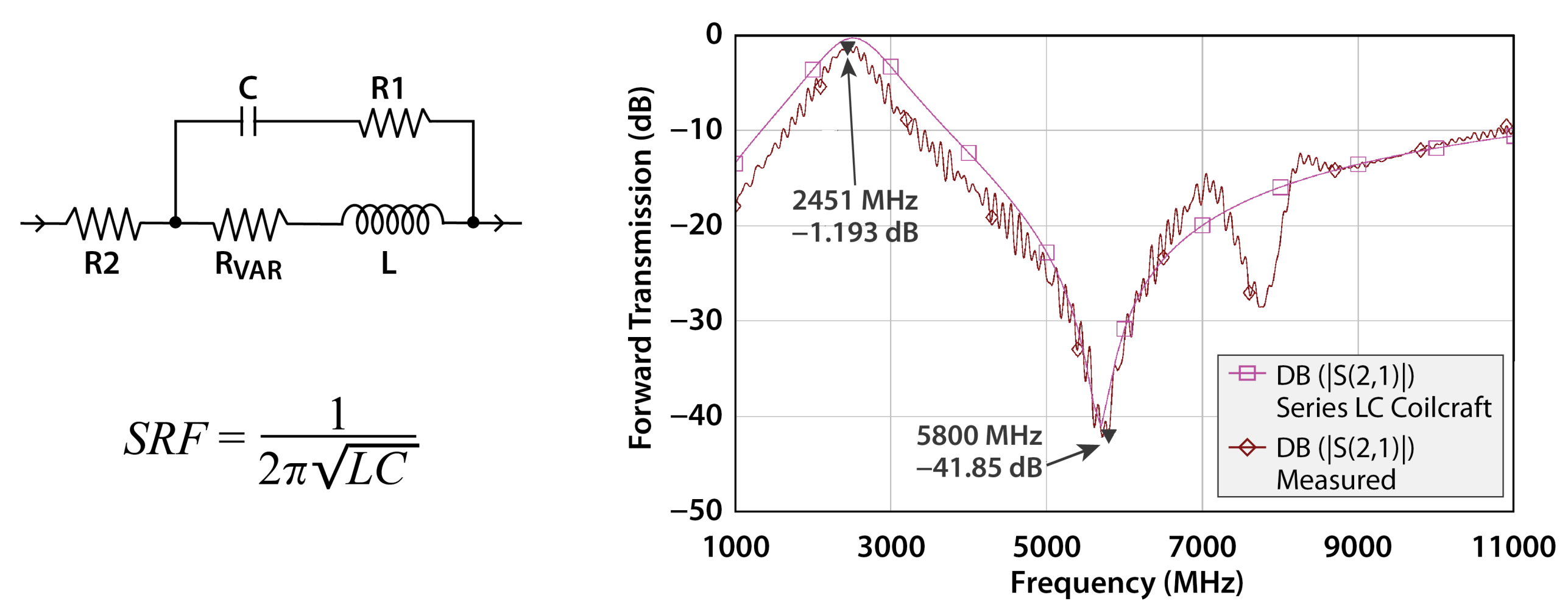

Only $0.0079 \%$ of the at $5.8 \mathrm{GHz}$. There is approximately $1 \mathrm{~dB}$
of attenuation at $2.45 \mathrm{GHz}$.

One can see the antenna is well matched to the system impedance by examining the reflection coefficient expressed in $\mathrm{dB}$ where over $95 \%$ of the power is delivered to the load at $2.45 \mathrm{GHz}$ and $5.8 \mathrm{GHz}$, corresponding to a voltage standing wave ratio of less than 1.4.

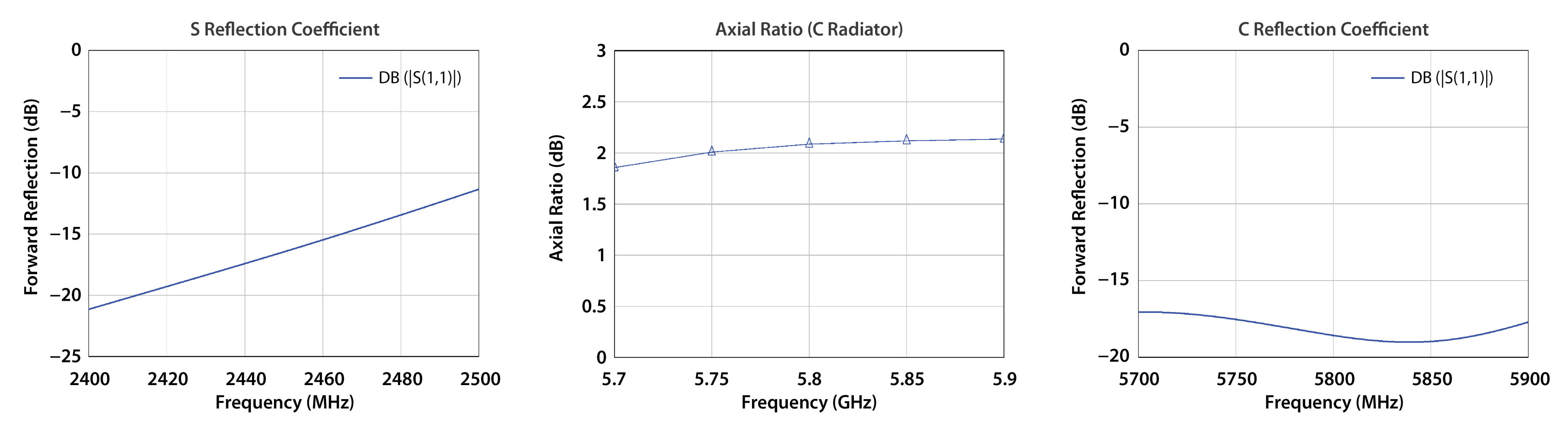

Simulated reflection

The simulated axial ratio, as seen above, is at an acceptable level below $3 \mathrm{~dB}$ for the $5.8 \mathrm{GHz}$ antenna; this confirms that the antenna is circularly polarized. The deviation from circular polarization is at most $2 \mathrm{~dB}$ over the frequency range of interest. Similar results are seen for the $\mathbf{S}$ band antenna. Furthermore, as seen in the measured results below, the divergence is $1.8 \mathrm{~dB}$ for the $\mathrm{S}$ band and $1.5 \mathrm{~dB}$ for the $\mathrm{C}$ band when rotating the antenna from a copolarization to cross-polarization orientation.

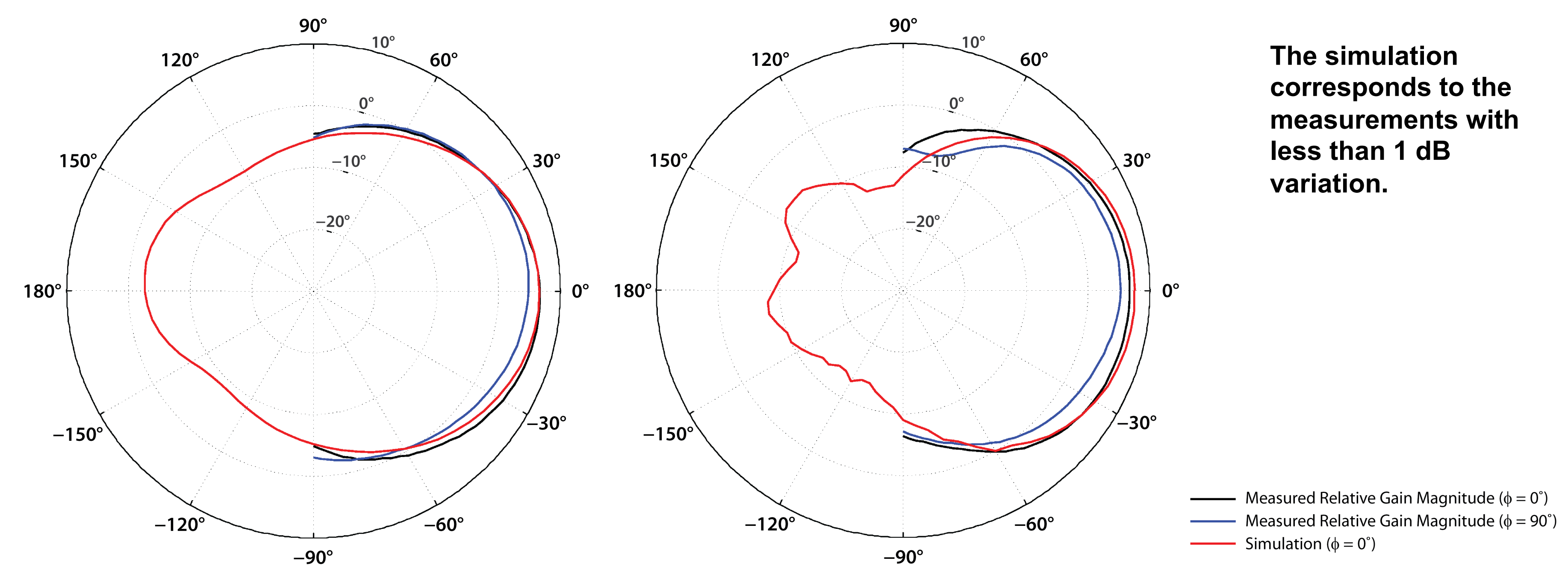

\section{Why is this important for our nation?}

Numerous DOE/DoD/OGA customers require the ability to detect microwave and radio frequency signals in specific frequency ranges. This approach will be adaptable to varying end uses by appropriately scaling the structure in frequency and/or increasing/decreasing the number of resonant elements as needed. This will result not only in a reduction in the system's size and weight, but also its cost.

It has been shown that coplanar elements can be collocated in the reactive near-field of another antenna and still provide excellent directivity and absolute gain in excess of $6 \mathrm{dBi}$, and maintain an acceptable impedance match. This is entirely necessary to provide the end user with tools capable of operation over various frequency ranges while maintaining a small package as nascent wireless technologies develop. A further investigation into the incorporation of additional 10.05 and $24.15 \mathrm{GHz}$ radiators in the same surface area is ongoing.

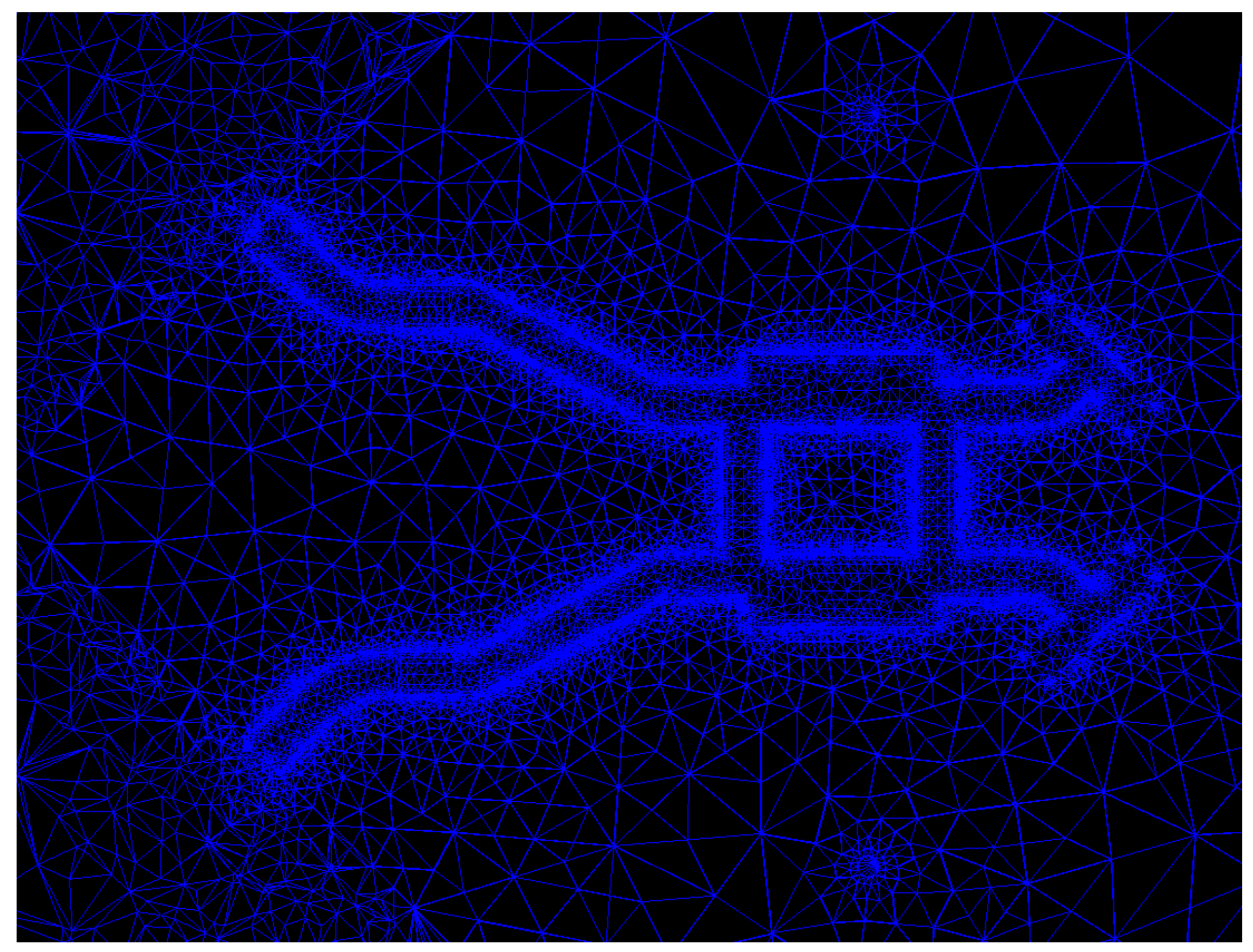

In the finite element magnetic radiation analysis, a combined form of Maxwell's equations is solved for throughout a compu-
tational volume that has been separated into 